\title{
Applying network analysis to investigate the links between dimensional schizotypy and cognitive and affective empathy
}

Yi Wang ${ }^{1,2}$, Hai-song Shi ${ }^{3}$, Wen-hua Liu ${ }^{4,5}$, Hong Zheng ${ }^{1,2}$, Keri Ka-Yee Wong ${ }^{6}$, Eric F. C. Cheung ${ }^{7}$, Raymond C. K. Chan ${ }^{1,2, *}$

1: Neuropsychology and Applied Cognitive Neuroscience Laboratory, CAS Key Laboratory of Mental Health, Institute of Psychology, Chinese Academy of Sciences, Beijing, China;

2: Department of Psychology, University of Chinese Academy of Sciences, Beijing, China;

3: North China Electric Power University, Beijing, China;

4: Department of Clinical Psychology, The Affiliated Brain Hospital of Guangzhou Medical University (Guangzhou Huiai Hospital), Guangzhou, China;

5: School of Health Management, Guangzhou Medical University, Guangzhou, China;

6: Department of Psychology \& Human Development, University College London, London, UK;

7: Castle Peak Hospital, Hong Kong Special Administrative Region, China

\footnotetext{
To whom correspondence should be addressed: Raymond Chan, Institute of Psychology, Chinese Academy of Sciences, 16 Lincui Road, Beijing 10101, China; Tel/Fax: 86-(0)10-64836274; E-mail: rckchan@psych.ac.cn.
} 


\title{
Applying network analysis to investigate the links between dimensional schizotypy and cognitive and affective empathy
}

\begin{abstract}
Background: Although impairment in empathy has been reported in schizophrenia spectrum disorders, little is known about the relationship between empathy and schizotypal traits. This study examines this relationship by applying network analysis to a large sample collected at 18-months follow-up in a longitudinal dataset.

Methods: One thousand four hundred and eighty-six college students were recruited and completed a set of self-reported questionnaires on empathy, schizotypy, depression, anxiety and stress. Networks were constructed by taking the subscale scores of these measures as nodes and partial correlations between each pair of nodes as edges. Network Comparison Tests were performed to investigate the differences between individuals with high and low schizotypy.

Results: Cognitive and affective empathy were strongly connected with negative schizotypy in the network. Physical and social anhedonia showed high centrality measured by strength, closeness and betweenness while anxiety and stress showed high expected influence. Predictability ranged from $22.4 \%$ (personal distress) to $79.9 \%$ (anxiety) with an average of $54.4 \%$. Compared with the low schizotypy group, the high schizotypy group showed higher global strength $(S=0.813, p<0.05)$ and significant differences in network structure $(M=0.531, p<0.001)$ and strength of edges connecting empathy with schizotypy (adjusted ps < 0.05).
\end{abstract}

Limitations: Only self-rating scales were used, and disorganized schizotypy was not included.

Conclusions: Our findings suggest that the cognitive and affective components of empathy and dimensions of schizotypy are closely related in the general population and their network interactions may play an important role in individuals with high schizotypy. 
Keywords: schizotypy; empathy; anhedonia; network analysis; centrality; network comparison 


\section{Introduction}

Empathy is a complex process that can be divided into at least two components: cognitive and affective empathy (Davis, 1983; Derntl et al., 2009; Reniers et al., 2011; Shamay-Tsoory et al., 2007). The cognitive component, likened to Theory of Mind (ToM), refers to an individual's ability to infer others' mental states or feelings; whereas affective empathy refers to the vicarious emotional experience of others (Reniers et al., 2011). Deficits in mentalizing ability have been reported in several mental disorders including schizophrenia spectrum disorders and mood disorders (Cotter et al., 2018). Two recent meta-analyses on empathy impairment in patients with schizophrenia found poorer cognitive and affective empathy as measured by self-reported or performance-based assessments (Bonfils et al., 2017, 2016). Bora et al. (2013) also found that patients with first-episode psychosis showed severe deficits in mentalizing ability compared with healthy controls (Cohen's $d=1.0)$. Furthermore, individuals with ultra-high risk for psychosis $(d=0.45)$ and unaffected relatives $(d=$ 0.37) of schizophrenia patients also exhibited impaired mentalizing ability (Bora and Pantelis, 2013).

Schizotypy is defined as a latent personality organization reflecting a putative liability for schizophrenia spectrum disorders (Meehl, 1962), which has been proposed as an approach to understand the development of psychosis (Kwapil and Barrantes-Vidal, 2015). Schizotypy consists of at least one positive (e.g., magical ideation, perceptual aberration) and one negative dimension (e.g., anhedonia) and its multidimensional structure has been validated recently in a cross-national study (Fonseca-Pedrero et al., 2018). Previous studies using neuropsychological and neuroimaging methods suggest that there are observable behavioural and brain changes associated with dimensional schizotypy (Nelson et al., 2013). As for empathy, a previous study using latent factor modelling to explore the association between dimensions of schizotypy and empathy found that negative schizotypy was negatively correlated with affective empathy (Bedwell et al., 2014). A recent study examining 
empathy in individuals with high levels of schizotypy found that individuals with positive schizotypy showed poorer performance on emotional perspective taking tasks than those with negative schizotypy and healthy controls; while both individuals with negative and positive schizotypy underperformed on affective responsiveness tasks compared with healthy controls (Pflum and Gooding, 2018). Although a number of studies have reported that the negative dimension of schizotypy may be correlated with empathy, particularly affective empathy (Henry et al., 2008; Wang et al., 2015, 2013), knowledge on the associations between dimensional schizotypy and cognitive and affective components of empathy remains limited. Furthermore, negative schizotypy is commonly associated with more negative affect, e.g. depression and anxiety (Debbané et al., 2009; Rey et al., 2009) and depression is reported to be associated with deficits of empathy (Derntl et al., 2012). In this study, we investigated how dimensional schizotypy, cognitive and affective components of empathy, and negative affect interact with each other using a network analysis.

A Network model assumes that symptoms and their interactions are the disorders themselves rather than reflections of the underlying common causes of mental disorders (Cramer et al., 2010). This novel approach takes psychological variables as nodes in a network and interactions between nodes as edges of the network. The key advantage of network analysis is that it allows for the examination of independent relationships between pairs of nodes while controlling for the effects of all other nodes in the network. Usually, to estimate a psychological network with continuous variables, partial correlations of each pair of nodes are calculated and the graphical Least Absolute Shrinkage and Selection Operator (LASSO) (Tibshirani, 1996) is applied to generate a sparse network with relatively strong connections. Hence, edges of an estimated network represent partial correlation coefficients between two nodes after controlling for the other nodes in the network (Epskamp et al., 2018). Furthermore, the importance of a node in the network could be assessed by measuring the centrality indices of the node, including betweenness, closeness and strength (Borsboom and Cramer, 2013). Betweenness and closeness are calculated 
based on the shortest path length between nodes. Nodes with high betweenness means that they appear in the shortest paths between other nodes frequently, while high closeness indicates close connections with all the other nodes in the network. The strength of a node is calculated by summing the absolute weights of all the edges connected to the node, suggesting how influential the node is in the network. Expected Influence (EI) assesses the strength of a node's influence within the network while accounting for the presence of negative edges (Robinaugh et al., 2016). In addition, the predictability of a node measures the degree to which a given node can be predicted by all remaining nodes in the network (Haslbeck \& Fried, 2017). The network model is particularly useful in improving our understanding of mental disorders by investigating the interactions between clinical symptoms and non-clinical features (Guloksuz et al., 2017; Isvoranu et al., 2017). For example, Fried et al. (2016) performed a network analysis in more than 3,000 patients with depression and found that both DSM (Diagnostic and Statistical Manual of Mental Disorders) and non-DSM symptoms were among the most central nodes in the network, suggesting that researchers and clinicians should focus more on these central symptoms and their interactions in future studies, especially in the assessment of treatment outcomes. To date, only a few studies have examined the network structure of empathy using the Interpersonal Reactivity Index (IRI) (Briganti et al., 2018), or network structure of schizotypy using the Schizotypal Personality Questionnaire (Dodell-Feder et al., 2019; E. Fonseca-Pedrero et al., 2018; Eduardo Fonseca-Pedrero et al., 2018), the Wisconsin Schizotypy Scales (Christensen et al., 2018), or the Oviedo Schizotypy Assessment Questionnaire (Fonseca-Pedrero et al., 2020). However, little is known about the network interactions between dimensional schizotypy, cognitive and affective empathy and negative affect in one network.

In this study, we performed network analysis using data collected at the most recent wave of an 18-month longitudinal follow-up study (Wang et al., 2018). Firstly, the network structure of the whole sample was estimated to examine the interactions of positive schizotypy (perceptual aberration and magical ideation), negative schizotypy (social anhedonia and physical anhedonia), cognitive empathy 
(perspective taking and fantasy subscales of the IRI), affective empathy (personal distress and empathic concern subscales of the IRI) and negative affect (depression, anxiety and stress). We hypothesized that negative schizotypy (social and physical anhedonia) would show strong associations with empathy, especially with affective component of empathy, after controlling for the other variables in the network. In addition, the centrality indices, El and predictability were calculated to identify the important nodes in the network. Similarly, we hypothesized that negative schizotypy and negative affect would be central nodes in the network based on their close relationships with other nodes reported in previous studies. Secondly, since there is significant gender bias in both empathy (Christov-Moore et al., 2014) and schizotypy (Eduardo Fonseca-Pedrero et al., 2018), the network structures for male and female participants were estimated separately and permutation tests were used to examine the invariance of network structure, global strength and edge-weights (van Borkulo et al., 2017). This comparison was used to examine whether the associations among dimensional schizotypy, cognitive and affective empathy and negative affect are similar for males and females. Lastly, we estimated and compared the networks between individuals with high and low levels of schizotypy. In our previous analysis we identified four latent classes with distinct trajectories in an 18-month follow-up study including the "low schizotypy" group (LC1), the "stable high schizotypy" group (LC3), the "high reactive schizotypy" group (LC2) and the "low reactive schizotypy" group (LC4) (Wang et al., 2018). At the last follow-up, the latter three latent groups showed higher level of schizotypy than LC1. Hence, we merged these three groups into a high schizotypy group (LC234) and constructed networks for the low schizotypy (LC1) and high schizotypy (LC234) groups separately and compared their differences in network structure, global strength and edge-weights. According to the hysteresis principle of the network theory (Borsboom, 2017) and previous studies using network comparison (van Rooijen et al., 2019), we assumed that the high schizotypy group would show a network with higher global strength compared with the low schizotypy group since their relatively high levels of negative affect and lower scores in cognitive and affective empathy may result in more connections and/or stronger 
correlations between nodes in the network.

\section{Materials and Methods}

\subsection{Participants}

The present sample was derived from the sample of a study we published previously on the 18-month longitudinal trajectory of schizotypal traits (Wang et al., 2018). The participating college students completed a 40-minute questionnaire session in a group at six-month intervals up to 18-months (four time points in total). Participants received monetary remuneration (about US\$2 for each session) in return for completing the questionnaires. In this study, we only used data from the last time point where empathy measurements were available for the whole sample. Fifty-five participants were further excluded because of missing values in the empathy measure, resulting in a final valid sample of 1486 participants (574 males, 912 females; mean age=20.2 years; $S D=1.1$ ) (see Table 1 for more details). Responders and non-responders did not differ in basic demographic information. This study was approved by the Ethics Committee of the Institute of Psychology, the Chinese Academy of Sciences. Written informed consent was obtained from all participants.

\subsection{Measures}

\subsubsection{Empathy}

The Interpersonal Reactivity Index (IRI) (Davis, 1983) is a self-reported scale measuring empathy and consists of four subscales: Perspective Taking (PT), Fantasy (FA), Personal Distress (PD) and Empathic Concern (EC). The first two subscales tap into the cognitive component of empathy, while the last two subscales capture affective empathy. The Chinese version of the IRI consisting of 22 items has been shown to have good reliability and validity in both patients with schizophrenia and the general population (Zhang et al., 2010). The Cronbach's alpha coefficient for the whole scale was 0.76 in the current sample. 


\subsubsection{Schizotypy}

Schizotypy was measured using the Chapman Psychosis Proneness Scales, including the Physical Anhedonia Scale, the Revised Social Anhedonia Scale, the Magical Ideation Scale and the Perceptual Aberration Scale (Chapman et al., 1980, 1978, 1976; Eckblad and Chapman, 1983). The Physical Anhedonia Scale was used to assess the inability to experience pleasure from typically pleasurable physical stimuli, while the Revised Social Anhedonia Scale was used to assess the inability to experience pleasure from social stimuli and social interactions. Both physical and social anhedonia scales were considered to measure the negative dimension of schizotypy, while the Magical Ideation Scale and the Perceptual Aberration Scale were considered to assess positive schizotypal traits (Kwapil et al., 2008). The Chinese versions of these four scales are psychometrically robust and have good reliability and validity (Chan et al., 2016, 2015).

\subsubsection{Depression, Anxiety and Stress}

The Depression Anxiety Stress Scale (DASS) (Lovibond and Lovibond, 1995) is a self-reported instrument designed to measure the negative emotional states of depression, anxiety and stress. The 21-item version of the DASS was used in this study to measure the severity of anxiety and depression symptoms. Participants were asked to use a four-point severity/frequency scale to rate the extent to which they had experienced each symptom over the past week from 'never' to 'most of the time'. The Chinese version has been previously shown to have good internal consistency and validity (Wang et al., 2016). The Cronbach's alpha of the scale was 0.95 in this study.

\subsection{Statistical analysis}

\subsubsection{Descriptive analysis}

The means, standard deviations and skewness of all measures, including the 
Chapman scales and subscales of the IRI, and the DASS are reported in Table 1. Since gender effects have been commonly reported for both schizotypy and empathy, we examined the gender differences on all measures using independent samples t tests. Differences between latent groups of high and low schizotypy (LC1 vs. LC234) on all measures were also analyzed using independent samples t tests. SPSS v19.0 was used and the significance level was set at $\alpha=.05$. Cohen's $d$ was calculated for effect size.

\subsubsection{Network construction and centrality estimation}

We first estimated a network using the whole sample, taking the IRI-PT, IRI-FA, IRI-PD and IRI-EC subscale scores, the depression, anxiety and stress subscale scores of the DASS-21, as well as the social anhedonia (RSAS), physical anhedonia (RPAS), magical ideation (MIS) and perceptual aberration (PAS) scale scores of the Chapman scales as nodes of the network. The edges of the network were partial correlations between each pair of nodes after controlling for all the other variables in the network. A Gaussian graphical model was estimated using graphical Least Absolute Shrinkage and Selection Operator (LASSO) (Tibshirani, 1996) in combination with Extended Bayesian Information Criteria (EBIC) model selection (Foygel and Drton, 2010). The tuning parameter, set as 0.5 (Beard et al., 2016; Foygel and Drton, 2010; Isvoranu et al., 2017), was applied to shrink the partial correlation coefficients and set small ones to zero to obtain a stable and interpretable network. The placement of the nodes in

the network was determined by the force-directed Fruchterman-Reingold algorithm (Fruchterman \& Reingold, 1991). In addition, the importance of each node in the network was further investigated by examining centrality indices, including strength, betweenness and closeness of nodes, as well as predictability and El. The standardized z scores of centrality indices were calculated. The "qgraph" and "mgm" packages (https://CRAN.R-project.org/package=qgraph) implemented in R statistical software (version 3.3.2, https://www.r-project.org/) were used for network construction, centrality calculation and visualization. 


\subsubsection{Network comparison between male and female participants}

In addition to estimating the network for the whole sample, we also estimated networks for male and female participants separately using a similar method as the network estimation of the whole sample. First, the invariance of network structure, global strength and edge-weights between the networks of male and female participants were tested using two-tailed permutation tests (10,000 times) (van Borkulo et al., 2017). The test for invariance of network structure depended on the value of the maximum difference between matrices consisting of all connection strengths. The global strength of networks, defined as the sum of the absolute connections of all pairs of nodes in the network was calculated and compared. False discovery rate (FDR) correction was adopted to address multiple comparisons of edge-weights, and adjusted $p$ values were calculated using the Benjamini and Hochberg (1995) method implemented in R. Furthermore, it is known that unequal group sizes may bias the result of network comparison tests and in this case subsampling from the larger group was recommended (van Borkulo et al., 2017). In this study, the sample sizes of male $(n=574)$ and female $(n=912)$ participants were different. As such, we also randomly selected a subsample of 574 female participants for network comparison with male participants (with 1,000 permutations) and this subsampling and comparison procedure was repeated 100 times. Proportions of $p<$ 0.05 for the invariance tests of network structure and global strength were reported. The "Network Comparison Test" package (https://CRAN.R-project.org/package=NetworkComparisonTest) implemented in R statistical software was used for the network comparisons. The significance threshold was set at $p$ or adjusted $p<0.05$.

\subsubsection{Network comparison between latent groups with high and low schizotypy}

In order to compare the network structures between latent groups with high and low schizotypy, we constructed separate networks for the two groups and carried out network comparison tests. Among the four latent groups, the "low schizotypy" group 
(LC1, $n=1079$ ) exhibited stable and low levels of schizotypal traits during follow-ups, the "stable high schizotypy" group $(\mathrm{LC} 3, \mathrm{n}=71)$ had persistently high levels of schizotypal traits during follow-ups, while the "high reactive schizotypy" group (LC2, $\mathrm{n}=136)$ and the "low reactive schizotypy" group (LC4, $\mathrm{n=200)}$ exhibited rapid or slow increment of schizotypal traits during follow-ups, and all of the latter three groups showed higher levels of schizotypy at the last time point than LC1. Considering the limited sample size of these three latent groups for network analysis, we merged participants from LC2, LC3 and LC4 into a high schizotypy group (LC234, n=407) and constructed networks for the LC1 and LC234 groups separately. First, network comparison test was performed to examine the invariance of network structure, global strength and edge strength using two-tailed permutation tests $(10,000$ times). In addition, in order to minimize sample size bias, a randomly selected subsample of 407 participants from the LC1 group was used for network comparison with the LC234 group (with 1,000 permutations). This subsampling and network comparison procedure was repeated 100 times and proportions of $p<0.05$ for the invariance tests of network structure and global strength were reported. The "Network Comparison Test" package (https://CRAN.R-project.org/package=NetworkComparisonTest) implemented in R statistical software was used. The significance threshold was set at $p$ or adjusted $p<$ 0.05 .

\subsubsection{Network stability and accuracy}

We calculated the stability of each estimated network, including the accuracy of edges and centrality stability according to a tutorial paper published by Epskamp, Borsboom and Fried (2016). First, the accuracy of edge-weights was estimated by drawing non-parametric bootstrapped Confidence Intervals (Cls) with 10000 permutations. Narrow bootstrapped Cls denoted low sampling variability in edge-weights, indicating that an accurate network was estimated. Then, we investigated the stability of the centrality indices using case-dropping subset 
bootstrap to assess how well the order of centralities was retained in different portions of data. The CS-coefficient was used to quantify this stability, which was the maximum drop in proportions to retain a correlation of 0.7 in at least $95 \%$ of the sample. It has been suggested that the CS-coefficient should not be below 0.25 and preferably above 0.5 in order to make a network stable and interpretable. Thirdly, we conducted bootstrapped difference tests between edge-weights and centrality indices of the nodes to test whether these differed significantly from each other. The "bootnet" package (https://CRAN.R-project.org/package=bootnet) implemented in R statistical software was used to estimate the network stability.

\section{Results}

\subsection{Network estimation for whole sample}

We estimated a regularized network including cognitive and affective empathy, dimensional schizotypy and negative affect, as shown in Figure 1. The internal connections of each scale were strong and showed communities of nodes especially for the DASS and the Chapman scales. At the same time, we observed significant negative connections between IRI-EC and social anhedonia, and between IRI-FA/IRI-PT and physical anhedonia. These negative links indicated that affective empathy was negatively correlated with social anhedonia after controlling for other variables in the network, while cognitive empathy was negatively correlated with physical anhedonia.

\subsection{Network inference}

The standardized centrality indices and El of each node are shown in Figure 1. Physical anhedonia and anxiety showed high strength, indicating strong connections with other nodes in the network. Physical anhedonia and social anhedonia showed high closeness, indicating short mean distance to other nodes; and high betweenness, indicating these nodes may function as bridges connecting different parts of the network. Anxiety and stress measured by the DASS showed high El in the network, 
indicating that negative affect were the most influential nodes when taking the negative edges into account. The predictability of nodes ranged from $22.4 \%$ (IRI-PD) to $79.9 \%$ (DASS anxiety) with a mean predictability of $54.4 \%$.

Insert Table 1 and Figure 1 About Here

\subsection{Network comparisons between male and female participants}

Independent sample $t$ tests showed a significant gender effect on schizotypy, empathy, and scores on the depression and anxiety subscales of the DASS (see Table 1). Compared with female participants, male participants showed higher levels of schizotypy, including social anhedonia, physical anhedonia, magical ideation and perceptual aberration; and lower scores on all subscales of the IRI and more severe depressive and anxiety symptoms measured by the DASS.

The regularized networks for male and female participants are shown in Figure 2. The test for invariance of network structure showed significant difference between network structures of male and female participants $(\mathrm{M}=0.175, p=0.016)$; and the invariance test of global strength revealed a marginally significant difference between networks of male and female participants $(S=0.701, p=0.051$; global strength for males' network is 5.52 and 4.82 for females' network). Regarding invariance of edge strengths, no significant difference was found after FDR correction. Repeated subsampling (100 times) from female participants revealed that the difference in network structure and global strength was significant in $51 \%$ and $20 \%$ of the invariance tests respectively. These findings indicated that the global strengths and edge strengths of networks for male and female participants were comparable.

Insert Figure 2 About Here

\subsection{Network comparisons between high and low schizotypy groups}

The descriptive information for the low schizotypy group (LC1) and the high schizotypy group (LC234) are shown in Table 2. Significant differences in most of the measures were found, indicating that the high schizotypy group not only showed 
higher levels of schizotypal traits, but also had poorer empathy and higher levels of depression, anxiety and stress compared with the low schizotypy group.

The regularized networks estimated for the two groups are shown in Figure 3. The network comparison tests showed significant difference in network structure ( $M$ $=0.531, p<0.001)$ and global strength $(S=0.813 ; p=0.028$; global strength for the LC1 group network was 4.35, and 5.16 for the LC234 group network), indicating that the LC234 group showed stronger connections across the whole network compared with the LC1 group. In addition, significant differences in edge strengths were observed after FDR correction, including IRI-PD - RPAS $(p<0.05)$, IRI-PD - MIS $(p<$ $0.05)$, IRI-PD - IRI-PT $(p<0.001)$, IRI-FA - RPAS $(p<0.05)$, IRI-FA - MIS $(p<0.05)$, RSAS - RPAS $(p<0.05)$, MIS - PAS $(p<0.001)$, RPAS - MIS $(p<0.05)$, and RPAS - DASS Stress $(p<0.001)$. Network comparisons based on repeated subsampling (100 times) from the LC1 group revealed that the difference was significant in $100 \%$ and $67 \%$ of invariance tests of network structure and global strength respectively.

Insert Figure 3 About Here

\subsection{Network stability and accuracy}

Stability analysis of the estimated network for the whole sample showed relatively narrow bootstrapped $\mathrm{Cls}$, suggesting reliable and accurate edge-weights. The CS-coefficient for strength $(\mathrm{CS}(\operatorname{cor}=0.7)=0.75)$ and edge-weights $(\mathrm{CS}(\operatorname{cor}=0.7)=$ 0.75 ) were larger than 0.5 , suggesting that the centrality indices were stable. The stability of the estimated networks for the LC1 and LC234 groups were examined separately. The bootstrapped Cls and CS-coefficient (LC1: CS (cor=0.7) for edge-weights was 0.75 , for strength was 0.75 ; LC234: CS (cor=0.7), for edge-weights was 0.75 , and for strength was 0.44 ) suggested accurate edges and relatively stable centrality indices in both networks. The stability of the estimated networks for male and female participants was also examined. The bootstrapped Cls and CS-coefficient (male: CS (cor=0.7) for edge-weights was 0.75, for strength was 0.67; female: CS (cor=0.7) for edge-weights was 0.75 , and for strength was 0.75 ) suggested accurate 
edges and stable centrality indices in the network. (See Supplementary Material for more details of the stability analysis).

\section{Discussion}

In this study, we examined the network interactions of cognitive and affective empathy, dimensional schizotypy and negative affect in a large sample of college students using a novel network approach. Taking all variables as nodes and partial correlations as edges between each pair of nodes, the estimated regularized network in the whole sample showed direct negative relationships between cognitive/affective empathy and negative schizotypy, specifically physical and social anhedonia, which is independent from the other variables in the network. Centrality analysis suggested that physical anhedonia and social anhedonia were important nodes with high centrality in the network, while negative affect showed high El in the network when taking the negative edges into account. The effect of gender on network invariance was investigated. Although significant differences in self-reported measures were found when we compared male and female participants, network comparison between male and female participants with repeated subsampling showed that the proportions of significant results were relatively low ( $20 \%$ for global strength and $51 \%$ for network structure). Most importantly, we compared the networks of a stable low schizotypy (LC1) group and a high schizotypy group (LC234) and found significant differences in network structure, global strength and edge-weights, indicating that the high schizotypy group had a more strongly connected network than the low schizotypy group. Significantly different edge-weights were observed in connections among cognitive empathy, positive schizotypy and IRI personal distress subscale scores, as well as the connection between negative and positive schizotypy.

Our findings of significant correlations between negative schizotypy and empathy are consistent with previous studies (Henry et al., 2008; Wang et al., 2015, 
2013). Bedwell and colleagues (2014) used both the Schizotypal Personality

Questionnaire and the Chapman scales to measure schizotypy and the Reading the Mind in the Eyes Test and the IRI to measure empathy. Using structural equation modelling, they found a negative relationship between the negative factor of schizotypy and empathy, but no significant relationship between empathy and other factors of schizotypy. Another study also reported no significant difference in empathy and theory of mind ability between individuals with high scores of magical ideation and controls (Canli et al., 2015). Hence, almost all previous studies suggest that the negative dimension of schizotypy appears to have an exclusive and clear relationship with empathy. In this study, the centrality analysis of the network in the whole sample also indicated that negative schizotypy is the most important node in the network, suggesting that changes in anhedonia may have significant effects on other nodes in the network, such as empathy.

In terms of the gender effect, we found significant differences between male and female participants on scores of empathy, anhedonia and negative affect based on independent samples t tests, which is consistent with previous studies (Christov-Moore et al., 2014; Eduardo Fonseca-Pedrero et al., 2018; Wang et al., 2016). When we compared the networks estimated for male and female participants, the invariance of both the global strength and edge strengths was non-significant. Although the invariance of network structure was significant, the repeated subsampling from female participants further indicated that in network comparisons with equal sample sizes, significant differences were only found in $20 \%$ of the invariance tests of global strength and $51 \%$ of the invariance tests of the network structure. These findings together indicate that although female participants had higher levels of empathy, lower levels of schizotypal traits and negative affect than male participants in general, male and female participants showed comparable network structure and similar patterns of pairwise interactions among empathy, schizotypy and negative affect.

Another interesting finding in the present study concerns the network comparison between the high schizotypy (LC234) and low schizotypy (LC1) groups, 
identified by latent class analysis in a previously published study (Wang et al., 2018). Four different latent groups with distinct trajectories were identified and we merged three of them into a high schizotypy group (LC234) since they all showed higher levels of schizotypy at the last time point. The high schizotypy group displayed poorer cognitive empathy, affective empathy and more severe depression, anxiety and stress compared with the low schizotypy (LC1) group. Network comparison revealed that the network of the high schizotypy group had greater global strength compared with the network of low schizotypy group. This finding is consistent with previous studies suggesting that individuals with more severe clinical symptoms would exhibit a more strongly connected network than individuals with mild clinical symptoms or individuals with subclinical symptoms in the general population (van Rooijen et al., 2018; Wigman et al., 2015). According to the hysteresis principle of network theory (Borsboom, 2017), if a symptom arises in a network that may influence other symptoms connected with it, then a set of closely connected symptoms may interact with each other and more symptoms may arise, resulting in a stronger connected network. In our case, compared with low schizotypy group, the high schizotypy group exhibited stronger connections among positive schizotypy, negative schizotypy and negative affect (including connections between magical ideation and perceptual aberration, between physical anhedonia and social anhedonia, and between physical anhedonia and magical ideation), suggesting that both positive and negative dimensions of schizotypy in this group might cause a spreading interaction of nodes in the network. At the same time, higher levels of negative schizotypy were associated with lower empathy, specifically affective and cognitive components of empathy which were closely linked with each other. This in turn, may enhance the feedback loop between negative schizotypy and empathy. The different network structures in the high schizotypy group in our study suggests that the interactions between negative schizotypy, positive schizotypy, empathy and negative affect may be feedback loops that play an important role in the changes of empathy in the high schizotypy group.

Our study has several limitations. First, disorganized schizotypy was considered 
as one dimension of schizotypy but not included in this study. Negative schizotypy was limited to anhedonia and other facets such as constricted affect was not included. Secondly, schizotypy, empathy and negative affect were measured by self-reported scales. As such, our results might have been biased by social desirability and recall bias. Recently, behavioural paradigms that capture different components of empathy, such as the Empathy Accuracy (Ripoll et al., 2013) and the Multidimensional Assessment of Empathic Ability (Derntl et al., 2010) have been developed. Future studies could make use of these novel paradigms to verify our findings. Thirdly, although good stability and edge-weight accuracy of the estimated network was noted in the whole sample, the network estimated for the latent groups may not be as stable because of the relatively small sample size. We have therefore merged three latent groups into a high schizotypy group in this study for network comparison tests after taking into consideration the sample size issue and hence we ignored the specific trajectories of each latent group during follow-ups. As there are concerns regarding the replicability and generalizability of network models (Forbes et al., 2017; Borsboom et al., 2017), future studies should replicate our findings and the inclusion of clinical samples may help to further elucidate the relationship between schizotypal traits and empathy. Lastly, the significant difference in network structure and edge-weights between the high and low schizotypy groups suggests that schizotypy may serve as a moderator in the network. Hence, a newly developed method such as the "Moderated Network Model" (Haslbeck et al., 2019) which extends the pairwise network model to a moderated network model could be used in the future.

To our knowledge, this is the first study to investigate the relationship among cognitive and affective components of empathy, positive and negative dimensions of schizotypy and negative affect using the network model. Our results suggest that the negative dimension of schizotypy may be directly related to cognitive and affective empathy, which is independent from other variables in the network, such as positive schizotypy and negative affect. Furthermore, individuals with high levels of schizotypal traits exhibited a more strongly connected network than those with low levels of schizotypal traits and connections between negative schizotypy and 
empathy may play an important role in the network. These findings provide evidence for a link between psychotic-like experiences and empathy and may have implications for early intervention and clinical practice. 


\section{Acknowledgement}

The authors have declared that there are no conflicts of interest in relation to the subject of this study. This work was supported by a grant from the National Science Fund China (31400884, 81571317), the Beijing Municipal Science \& Technology Commission Grant (Z161100000216138), the Beijing Training Project for the Leading Talents in Science and Technology (Z151100000315020), CAS Key Laboratory of Mental Health, Institute of Psychology; and China Scholarship Council. 


\section{References}

Beard, C., Millner, A.J., Forgeard, M.J.C., Fried, E.I., Hsu, K.J., Treadway, M.T., ... Björgvinsson, T. (2016). Network analysis of depression and anxiety symptom relationships in a psychiatric sample. Psychological Medicine, 46, 3359-3369. doi: 10.1017/S0033291716002300

Bedwell, J.S., Compton, M.T., Jentsch, F.G., Deptula, A.E., Goulding, S.M., Tone, E.B. (2014). Latent factor modeling of four schizotypy dimensions with theory of mind and empathy. PLoS One, 9, e113853. doi: 10.1371/journal.pone.0113853

Benjamini, Y., \& Hochberg, Y. (1995). Controlling the False Discovery Rate: A Practical and Powerful Approach to Multiple Testing. Journal of the Royal Statistical Society. Series B (Methodological), 57, 289-300.

Bonfils, K.A., Lysaker, P.H., Minor, K.S., Salyers, M.P. (2017). Empathy in schizophrenia: A meta-analysis of the Interpersonal Reactivity Index. Psychiatry Research, 249, 293-303. doi: 10.1016/j.psychres.2016.12.033

Bonfils, K.A., Lysaker, P.H., Minor, K.S., Salyers, M.P. (2016). Affective empathy in schizophrenia: a meta-analysis. Schizophrenia Research, 175, 109-117. doi: 10.1016/j.schres.2016.03.037

Bora, E., Pantelis, C. (2013). Theory of mind impairments in first-episode psychosis, individuals at ultra-high risk for psychosis and in first-degree relatives of schizophrenia: Systematic review and meta-analysis. Schizophrenia Research, 144, 31-36. doi: 10.1016/j.schres.2012.12.013

Borsboom, D. (2017). A network theory of mental disorders. World Psychiatry, 16, 5-13. doi: 10.1002/wps.20375

Borsboom, D., Cramer, A.O.J. (2013). Network Analysis: An Integrative Approach to the Structure of Psychopathology. Annual Review of Clinical Psychology, 9, 91-121. doi: 10.1146/annurev-clinpsy-050212-185608

Borsboom, D., Fried, E. I., Epskamp, S., Waldorp, L. J., van Borkulo, C. D., van der Maas, H. L. J., \& Cramer, A. O. J. (2017). False alarm? A comprehensive reanalysis of 'Evidence that psychopathology symptom networks have limited replicability' by Forbes, Wright, Markon, and Krueger (2017). Journal of Abnormal Psychology, 126, 989-999. doi: 10.1037/abn0000306

Briganti, G., Kempenaers, C., Braun, S., Fried, E.I., Linkowski, P. (2018). Network analysis of empathy items from the interpersonal reactivity index in 1973 young adults. Psychiatry Research, 265, 87-92. doi: 10.1016/j.psychres.2018.03.082

Canli, D., Ozdemir, H., Kocak, O.M. (2015). Magical ideation associated social cognition in adolescents: signs of a negative facial affect recognition deficit. Comprehensive Psychiatry, 61, 90-96. doi: 10.1016/j.comppsych.2015.05.009

Chan, R.C.K., Gooding, D.C., Shi, H.S., Geng, F.L., Xie, D.J., Yang, Z.Y., ... Cheung, E.F.C. (2016). Evidence of structural invariance across three groups of Meehlian schizotypes. npj Schizophrenia, 2, 16016. doi: 10.1038/npjschz.2016.16

Chan, R.C.K., Shi, H.S., Geng, F.L., Liu, W.H., Yan, C., Wang, Y., Gooding, D.C. (2015). 
The Chapman psychosis-proneness scales: Consistency across culture and time. Psychiatry Research, 228, 143-149. doi: 10.1016/j.psychres.2015.04.031

Chapman, L.J., Chapman, J.P., Raulin, M.L. (1978). Body-image aberration in schizophrenia. Journal of Abnormal Psychology, 87, 399-407. doi: 10.1037/0021-843X.87.4.399

Chapman, L.J., Chapman, J.P., Raulin, M.L. (1976). Scales for physical and social anhedonia. Journal of Abnormal Psychology, 85, 374-382. doi: 10.1037/0021-843X.85.4.374

Chapman, L.J., Edell, W.S., Chapman, J.P. (1980). Physical anhedonia, perceptual aberration, and psychosis proneness. Schizophrenia Bulletin, 6, 639-653. doi: $10.1093 /$ schbul/6.4.639

Christensen, A.P., Kenett, Y.N., Aste, T., Silvia, P.J., \& Kwapil, T.R. (2018). Network structure of the Wisconsin Schizotypy Scales-Short Forms: Examining psychometric network filtering approaches. Behavior Research Methods, 50, 2531-2550. doi: 10.3758/s13428-018-1032-9

Christov-Moore, L., Simpson, E.A., Coudé, G., Grigaityte, K., lacoboni, M., Ferrari, P.F. (2014). Empathy: Gender effects in brain and behavior. Neuroscience and Biobehavioral Review, 46, 604-627. doi: 10.1016/j.neubiorev.2014.09.001

Cotter, J., Granger, K., Backx, R., Hobbs, M., Looi, C.Y., Barnett, J.H. (2018). Social cognitive dysfunction as a clinical marker: A systematic review of meta-analyses across 30 clinical conditions. Neuroscience and Biobehavioral Review, 84, 92-99. doi: 10.1016/j.neubiorev.2017.11.014

Cramer, A.O.J., Waldorp, L.J., Van Der Maas, H.L.J., Borsboom, D. (2010). Comorbidity: A network perspective. The Behavioral and Brain Sciences, 33, 137-150. doi: 10.1017/S0140525X09991567

Davis, M.H. (1983). Measuring individual differences in empathy: Evidence for a multidimensional approach. Journal of Personality and Social Psychology, 44, 113-126. doi: 10.1037/0022-3514.44.1.113

Debbané, M., Van Der Linden, M., Gex-Fabry, M., Eliez, S. (2009). Cognitive and emotional associations to positive schizotypy during adolescence. Journal of Child Psychology and Psychiatry, and Allied Disciplines, 50, 326-334. doi: 10.1111/j.1469-7610.2008.01961.x

Derntl, B., Finkelmeyer, A., Eickhoff, S., Kellermann, T., Falkenberg, D.I., Schneider, F., Habel, U. (2010). Multidimensional assessment of empathic abilities: Neural correlates and gender differences. Psychoneuroendocrinology, 35, 67-82. doi: 10.1016/j.psyneuen.2009.10.006

Derntl, B., Finkelmeyer, A., Toygar, T.K., Hülsmann, A., Schneider, F., Falkenberg, D.I., Habel, U. (2009). Generalized deficit in all core components of empathy in schizophrenia. Schizophrenia Research, 108, 197-206. doi: 10.1016/j.schres.2008.11.009

Derntl, B., Seidel, E.M., Schneider, F., Habel, U. (2012). How specific are emotional deficits? A comparison of empathic abilities in schizophrenia, bipolar and depressed patients. Schizophrenia Research, 142, 58-64. doi: 10.1016/j.schres.2012.09.020 
Dodell-Feder, D., Saxena, A., Rutter, L., Germine, L. (2019). The network structure of schizotypal personality traits in a population-based sample. Schizophrenia Research, 208, 258-267. doi: 10.1016/j.schres.2019.01.046

Eckblad, M., Chapman, L.J. (1983). Magical ideation as an indicator of schizotypy. Journal of Consulting and Clinical Psychology, 51, 215-225. doi: 10.1037/0022-006X.51.2.215

Epskamp, S., Borsboom, D., Fried, E.I. (2018). Estimating Psychological Networks and their Accuracy: A Tutorial Paper. Behavior Research Methods, 50, 195-212. doi: 10.3758/s13428-017-0862-1

Fonseca-Pedrero, E., Chan, R.C.K., Debbané, M., Cicero, D., Zhang, L.C., Brenner, C., ... Ortuño-Sierra, J. (2018). Comparisons of schizotypal traits across 12 countries: Results from the International Consortium for Schizotypy Research. Schizophrenia Research, 199, 128-134. doi: 10.1016/j.schres.2018.03.021

Fonseca-Pedrero, E., Debbané, M., Ortuño-Sierra, J., Chan, R.C.K., Cicero, D.C., Zhang, L.C., ... Jablensky, A. (2018). The structure of schizotypal personality traits: A cross-national study. Psychological Medicine, 48, 451-462. doi: 10.1017/S0033291717001829

Fonseca-Pedrero, Eduardo, Ortuño, J., Debbané, M., Chan, R. C. K., Cicero, D., Zhang, L. C., Brenner, C., Barkus, E., Linscott, R. J., Kwapil, T., Barrantes-Vidal, N., Cohen, A., Raine, A., Compton, M. T., Tone, E. B., Suhr, J., Inchausti, F., Bobes, J., Fumero, A., ... Fried, E. I. (2018). The Network Structure of Schizotypal Personality Traits. Schizophrenia Bulletin, 44(suppl_2), S468-S479. doi: 10.1093/schbul/sby044

Fonseca-Pedrero, E., Ortuno-Sierra, J., Inchausti, F., Rodriguez-Testal, J. F., \& Debbane, M. (2020). Beyond Clinical High-Risk State for Psychosis: The Network Structure of Multidimensional Psychosis Liability in Adolescents. Frontiers in Psychiatry, 10, 967. doi: 10.3389/fpsyt.2019.00967

Forbes, M. K., Wright, A. G. C., Markon, K. E., \& Krueger, R. F. (2017). Evidence that psychopathology symptom networks have limited replicability. Journal of Abnormal Psychology, 126, 969-988. doi: 10.1037/abn0000276

Foygel, R., Drton, M. (2010). Extended Bayesian information criteria for Gaussian graphical models, in: Advances in Neural Information Processing Systems 23: 24th Annual Conference on Neural Information Processing Systems 2010, NIPS 2010. pp. 604-612.

Fried, E. I., Epskamp, S., Nesse, R. M., Tuerlinckx, F., \& Borsboom, D. (2016). What are "good" depression symptoms? Comparing the centrality of DSM and non-DSM symptoms of depression in a network analysis. Journal of Affective Disorders, 189, 314-320. doi: 10.1016/j.jad.2015.09.005

Fruchterman, T. M., \& Reingold, E. M. (1991). Graph drawing by force-directed placement. Software: Practice and experience, 21, 1129-1164.

Guloksuz, S., Pries, L.K., Van Os, J. (2017). Application of network methods for understanding mental disorders: Pitfalls and promise. Psychological Medicine, 47, 2743-2752. doi: 10.1017/S0033291717001350

Haslbeck, J.M.B., Borsboom, D., \& Waldorp, L.J. (2019). Moderated Network Models. Multivariate Behavioral Research, 0(0), 1-32. doi: 


\subsection{0/00273171.2019.1677207}

Haslbeck, J.M.B., \& Waldorp, L.J. (2018). How well do network models predict observations? On the importance of predictability in network models. Behavior Research Methods, 50, 853-861. doi: 10.3758/s13428-017-0910-x

Henry, J.D., Bailey, P.E., Rendell, P.G. (2008). Empathy, social functioning and schizotypy. Psychiatry Research, 160, 15-22. doi:

10.1016/j.psychres.2007.04.014

Isvoranu, A.M., van Borkulo, C.D., Boyette, L.L., Wigman, J.T.W., Vinkers, C.H., Borsboom, D., ... Myin-Germeys, I. (2017). A network approach to psychosis: Pathways between childhood trauma and psychotic symptoms. Schizophrenia Bulletin, 43, 187-196. doi: 10.1093/schbul/sbw055

Kwapil, T.R., Barrantes-Vidal, N. (2015). Schizotypy: Looking back and moving forward. Schizophrenia Bulletin, 41, S366-S373. doi: 10.1093/schbul/sbu186

Kwapil, T.R., Barrantes-Vidal, N., Silvia, P.J. (2008). The dimensional structure of the wisconsin schizotypy scales: Factor identification and construct validity. Schizophrenia Bulletin, 34, 444-457. doi: 10.1093/schbul/sbm098

Lovibond, P.F., Lovibond, S.H. (1995). The structure of negative emotional states: Comparison of the Depression Anxiety Stress Scales (DASS) with the Beck Depression and Anxiety Inventories. Behaviour Research and Therapy, 33, 335-343. doi: 10.1016/0005-7967(94)00075-U

Meehl, P.E. (1962). Schizotaxia, schizotypy, schizophrenia. The American Psychologist, 17, 827-838. doi: 10.1037/h0041029

Nelson, M.T., Seal, M.L., Pantelis, C., Phillips, L.J. (2013). Evidence of a dimensional relationship between schizotypy and schizophrenia: A systematic review. Neuroscience and Biobehavioral Reviews, 37, 317-327. doi: 10.1016/j.neubiorev.2013.01.004

Pflum, M.J., Gooding, D.C. (2018). Context matters: Social cognition task performance in psychometric schizotypes. Psychiatry Research, 264, 398-403. doi: 10.1016/j.psychres.2018.03.075

Raine, A. (1991). The SPQ: A scale for the assessment of schizotypal personality based on DSM-III-R criteria. Schizophrenia Bulletin, 17, 555-564. doi: 10.1093/schbul/17.4.555

Reniers, R.L., Corcoran, R., Drake, R., Shryane, N.M., Völlm, B.A. (2011). The QCAE: A Questionnaire of Cognitive and Affective Empathy. Journal of Personality Assessment, 93, 84-95. doi: 10.1080/00223891.2010.528484

Rey, G., Jouvent, R., Dubal, S. (2009). Schizotypy, depression, and anxiety in physical and social anhedonia. Journal of Clinical Psychology, 65, 695-708. doi: 10.1002/jclp.20577

Ripoll, L.H., Zaki, J., Perez-Rodriguez, M.M., Snyder, R., Strike, K.S., Boussi, A., ... New, A.S. (2013). Empathic accuracy and cognition in schizotypal personality disorder. Psychiatry Res. 210, 232-241. doi: 10.1016/j.psychres.2013.05.025

Robinaugh, D.J., Millner, A.J., \& McNally, R.J. (2016). Identifying highly influential nodes in the complicated grief network. Journal of Abnormal Psychology, 125, 747-757. doi:10.1037/abn0000181 
Shamay-Tsoory, S.G., Shur, S., Barcai-Goodman, L., Medlovich, S., Harari, H., Levkovitz, Y. (2007). Dissociation of cognitive from affective components of theory of mind in schizophrenia. Psychiatry Research, 149, 11-23. doi: 10.1016/j.psychres.2005.10.018

Tibshirani, R. (1996). Regression Shrinkage and Selection Via the Lasso. Journal of the Royal Statistical Society. Series B (Methodological), 58, 267-288. doi: 10.1111/j.2517-6161.1996.tb02080.x

van Borkulo, C.D., Boschloo, L., Kossakowski, J.J., Tio, P., Schoevers, R.A., Borsboom, D., Waldorp, L.J. (2017). Comparing network structures on three aspects: A permutation test. doi: 10.13140/RG.2.2.29455.38569

van Rooijen, G., Isvoranu, A.M., Kruijt, O.H., van Borkulo, C.D., Meijer, C.J., Wigman, J.T.W., ... Bartels-Velthuis, A.A. (2018). A state-independent network of depressive, negative and positive symptoms in male patients with schizophrenia spectrum disorders. Schizophrenia Research, 193, 232-239. doi: 10.1016/j.schres.2017.07.035

Wang, K., Shi, H.S., Geng, F.L., Zou, L.Q., Tan, S.P., Wang, Y., ... Chan, R.C.K. (2016). Cross-cultural validation of the Depression Anxiety Stress Scale-21 in China. Psychological Assessment, 28, e88-e100. doi: 10.1037/pas0000207

Wang, Y., Liu, W.H., Li, Z., Wei, X.H., Jiang, X.Q., Neumann, D.L., Chan, R.C.K. (2015). Dimensional schizotypy and social cognition: An fMRI imaging study. Frontiers of Behaviour Neuroscience, 9, 133. doi: 10.3389/fnbeh.2015.00133

Wang, Y., Neumann, D.L., Shum, D.H.K., Liu, W.H., Shi, H.S., Yan, C., ... Chan, R.C.K. (2013). Cognitive empathy partially mediates the association between negative schizotypy traits and social functioning. Psychiatry Research, 210, 62-68. doi: 10.1016/j.psychres.2013.03.015

Wang, Y, Shi, H.S., Liu, W.H., Xie, D.J., Geng, F.L., Yan, C., ... Chan, R.C.K. (2018). Trajectories of schizotypy and their emotional and social functioning: An 18-month follow-up study. Schizophrenia Research, 193, 384-390. doi: 10.1016/j.schres.2017.07.038

Wigman, J.T.W., Van Os, J., Borsboom, D., Wardenaar, K.J., Epskamp, S., Klippel, A., Viechtbauer, W., Myin-Germeys, I., Wichers, M. (2015). Exploring the underlying structure of mental disorders: Cross-diagnostic differences and similarities from a network perspective using both a top-down and a bottom-up approach. Psychological Medicine, 45, 2375-2387. doi: 10.1017/S0033291715000331

Zhang, F., Dong, Y., Wang, K. (2010). Reliability and validity of the Chinese version of the Interpersonal Reactivity Index-C. Chinese Journal of Clinical Psychology, 18, 155-157. doi: 10.16128/j.cnki.1005-3611.2010.02.019 


\section{Figure Legends}

Figure 1. Estimated regularized network structure of empathy, schizotypy and depression, anxiety and stress in the whole sample (left) and the centrality indices of nodes in the network (right). The green edges indicate positive partial correlations and edges in red indicates negative partial correlations. Thicker lines represent stronger connections. The blue ring around each node represents its predictability values. Centrality indices and Expected Influence are shown as standardized z-scores. RSAS: Chinese Version of Revised Social Anhedonia Scale; RPAS: Chinese Version of Physical Anhedonia Scale; MIS: Chinese Version of Magical Ideation Scale; PAS: Chinese Version of Perceptual Aberration Scale; IRI: Interpersonal Reactivity Index; PT: Perspective Taking; FA: Fantasy; EC: Empathic Concern; PD: Personal Distress; DASS: Depression Anxiety Stress Scale-21item.

Figure 2. Estimated regularized networks in male (left, $n=574$ ) and female (right, $\mathbf{n = 9 1 2 )}$ participants. The green edges indicate positive partial correlations and edges in red indicate negative partial correlations. Thicker lines represent stronger connections. The blue ring around each node represents its predictability values. RSAS: Chinese Version of Revised Social Anhedonia Scale; RPAS: Chinese Version of Physical Anhedonia Scale; MIS: Chinese Version of Magical Ideation Scale; PAS: Chinese Version of Perceptual Aberration Scale; IRI: Interpersonal Reactivity Index; PT: Perspective Taking; FA: Fantasy; EC: Empathic Concern; PD: Personal Distress; DASS: Depression Anxiety Stress Scale-21item.

Figure 3. Estimated regularized networks for low schizotypy group (LC1) (left, $\mathrm{n}=1079$ ) and high schizotypy group (LC234) (right, $n=407$ ). The green edges indicate positive correlations and edges in red indicate negative correlations. Thicker lines represent stronger connections. Numbers on lines showed the absolute values of regularized partial correlation coefficients. The blue ring around each node represents its predictability values. RSAS: Chinese Version of Revised Social Anhedonia Scale; RPAS: Chinese Version of Physical Anhedonia Scale; MIS: Chinese Version of Magical Ideation Scale; PAS: Chinese Version of Perceptual Aberration Scale; IRI: Interpersonal Reactivity Index; PT: Perspective Taking; FA: Fantasy; EC: Empathic Concern; PD: Personal Distress; DASS: Depression Anxiety Stress Scale-21item. 
Tables

Table 1. Descriptive analysis in the whole sample and gender effect

\begin{tabular}{|c|c|c|c|c|c|c|c|c|}
\hline & \multicolumn{3}{|c|}{$\begin{array}{l}\text { whole sample } \\
\qquad(n=1486)\end{array}$} & \multicolumn{2}{|c|}{$\begin{array}{c}\text { male } \\
(n=574)\end{array}$} & \multicolumn{2}{|c|}{$\begin{array}{l}\text { female } \\
(n=912)\end{array}$} & \multirow{2}{*}{$\begin{array}{l}\text { gender effect } \\
(t, \text { Cohen's } d)\end{array}$} \\
\hline & mean & S.D. & skewness & mean & S.D. & mean & S.D. & \\
\hline CSAS & 8.90 & 6.35 & 0.782 & 10.67 & 6.90 & 7.78 & 5.70 & $8.39^{* * *}, 0.46$ \\
\hline CPAS & 13.91 & 9.79 & 0.731 & 16.26 & 11.24 & 12.43 & 8.44 & $7.01^{* * *}, 0.39$ \\
\hline MIS & 10.19 & 5.15 & 0.787 & 11.01 & 5.52 & 9.67 & 4.83 & $4.79^{* * *}, 0.26$ \\
\hline PAS & 6.37 & 7.25 & 1.866 & 8.49 & 8.71 & 5.04 & 5.77 & $8.40^{* * *}, 0.48$ \\
\hline IRI_PT & 3.44 & 0.69 & -0.619 & 3.29 & 0.73 & 3.53 & 0.66 & $-6.44^{* * *},-0.35$ \\
\hline IRI_FA & 3.44 & 0.57 & 0.057 & 3.27 & 0.52 & 3.54 & 0.57 & $-9.25^{* * *},-0.48$ \\
\hline IRI_EC & 3.55 & 0.53 & -0.056 & 3.42 & 0.50 & 3.63 & 0.53 & $-7.77^{* * *},-0.41$ \\
\hline IRI_PD & 2.71 & 0.72 & -0.267 & 2.55 & 0.69 & 2.81 & 0.72 & $-7.03^{* * *},-0.37$ \\
\hline DASS_Depression & 2.24 & 3.30 & 1.95 & 2.87 & 3.97 & 1.84 & 2.73 & $5.43^{* * *}, 0.31$ \\
\hline DASS_Anxiety & 2.81 & 3.41 & 1.58 & 3.21 & 3.91 & 2.56 & 3.02 & $3.41^{* *}, 0.19$ \\
\hline DASS_Stress & 3.44 & 3.70 & 1.18 & 3.61 & 3.89 & 3.34 & 3.58 & $1.36,0.07$ \\
\hline
\end{tabular}

Note. CSAS: Chinese Version of Revised Social Anhedonia Scale; CPAS: Chinese Version of Physical Anhedonia Scale; MIS: Chinese Version of Magical Ideation Scale; PAS: Chinese Version of Perceptual Aberration Scale; IRI: Interpersonal Reactivity Index; PT: Perspective Taking; FA: Fantasy; EC: Empathic Concern; PD: Personal Distress; DASS: Depression Anxiety Stress Scale-21item. Independent sample t tests were performed for the gender effects. 
Table 2. Differences between latent groups of high and low schizotypy

\begin{tabular}{|c|c|c|c|c|c|}
\hline & \multicolumn{2}{|c|}{$\begin{array}{c}\text { LC1 } \\
(n=1079)\end{array}$} & \multicolumn{2}{|c|}{$\begin{array}{c}\text { LC234 } \\
(n=407)\end{array}$} & \multirow{2}{*}{$\begin{array}{l}\text { Group effect } \\
(t, \text { Cohen's } d)\end{array}$} \\
\hline & mean & S.D. & mean & S.D. & \\
\hline CSAS & 6.04 & 3.87 & 16.48 & 5.30 & $36.26^{* * *},-1.65$ \\
\hline CPAS & 9.39 & 5.87 & 25.90 & 7.80 & $38.76^{* * *},-1.69$ \\
\hline MIS & 8.45 & 3.87 & 14.78 & 5.30 & $21.98^{* * *},-1.23$ \\
\hline PAS & 3.27 & 2.75 & 14.59 & 8.87 & $25.28^{* * *},-1.56$ \\
\hline IRI_PT & 3.61 & 0.59 & 3.00 & 0.75 & $-14.60^{* * *}, 0.87$ \\
\hline IRI_FA & 3.56 & 0.53 & 3.11 & 0.52 & $-14.35^{* * *}, 0.78$ \\
\hline IRI_EC & 3.70 & 0.48 & 3.14 & 0.42 & $-21.88^{* * *}, 1.05$ \\
\hline IRI_PD & 2.67 & 0.71 & 2.83 & 0.73 & $3.81^{* * *},-0.22$ \\
\hline DASS_Depression & 1.28 & 2.05 & 4.79 & 4.44 & $15.34^{* * *},-1.06$ \\
\hline DASS_Anxiety & 1.91 & 2.39 & 5.19 & 4.42 & $14.18^{* * *},-0.96$ \\
\hline DASS_Stress & 2.69 & 3.12 & 5.46 & 4.32 & $11.82^{* * *},-0.75$ \\
\hline
\end{tabular}

Note. CSAS: Chinese Version of Revised Social Anhedonia Scale; CPAS: Chinese Version of Physical Anhedonia Scale; MIS: Chinese Version of Magical Ideation Scale; PAS: Chinese Version of Perceptual Aberration Scale; IRI: Interpersonal Reactivity Index; PT: Perspective Taking; FA: Fantasy; EC: Empathic Concern; PD: Personal Distress; DASS: Depression Anxiety Stress Scale-21item. 



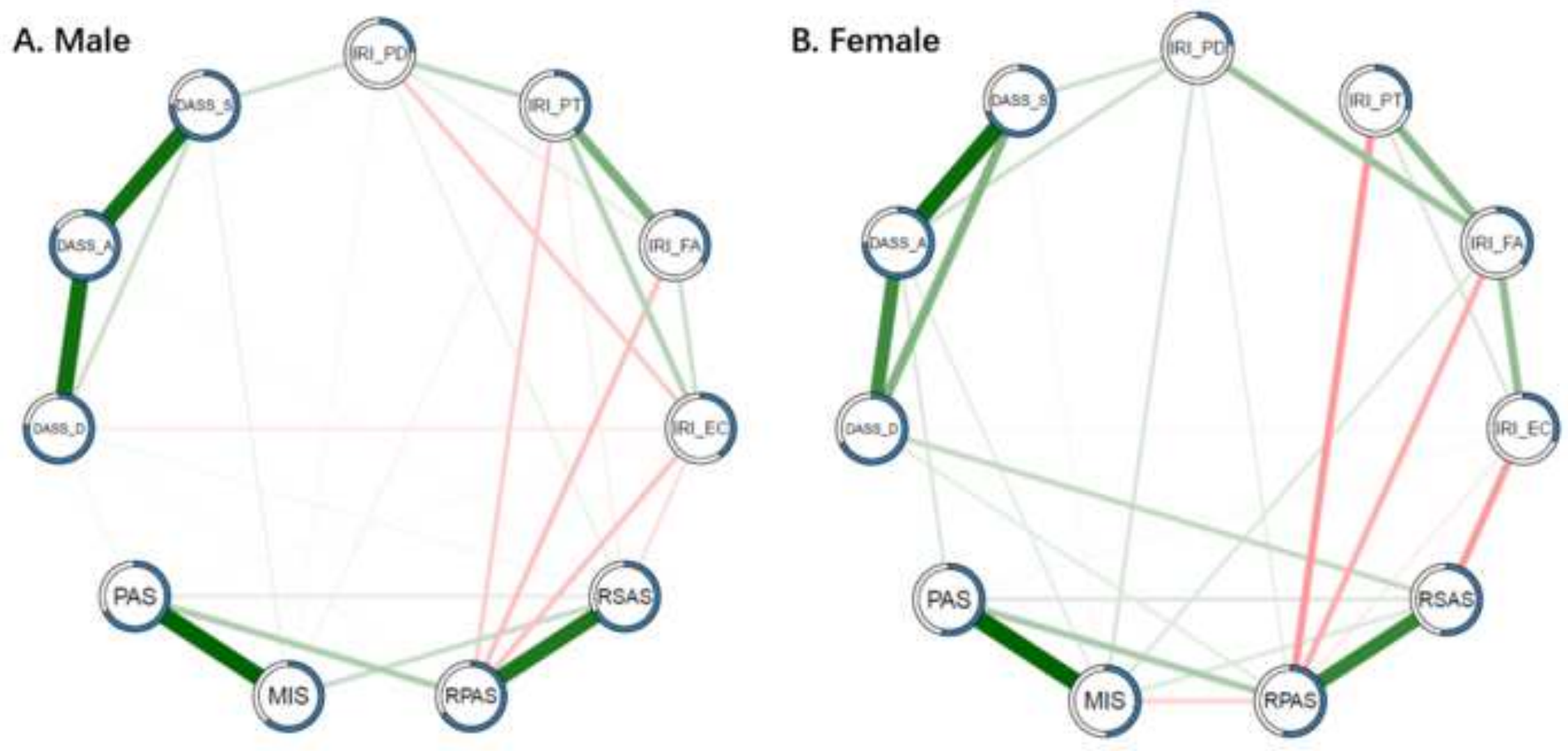

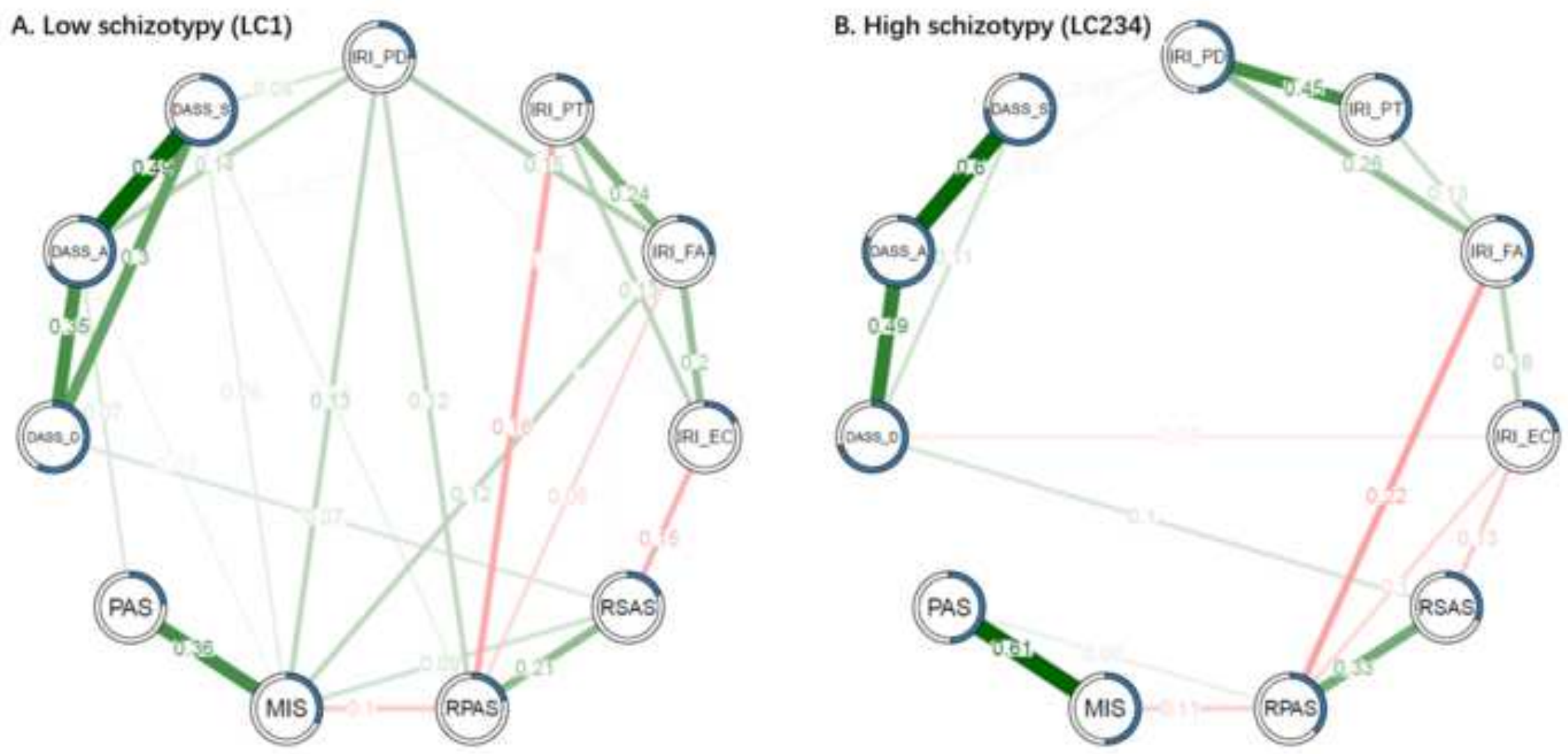
Click here to access/download RDM Data Profile XML DataProfile_4996996.xml 


\section{Declarations of interest:}

None. 


\section{Contributors}

Author RCK designed the study and wrote the protocol. Authors HSS and WHL collected the data, analyzed and interpreted the data. Author WY managed the literature searches, undertook the statistical analysis, and wrote the first draft of the manuscript. Authors KKYW, EFCC, CCP, YTM commented critically to the manuscript. All authors contributed to and have approved the final manuscript.

\section{Role of the funding source}

The funding agents had no further role in the study design; in the collection, analysis and interpretation of the data; in the writing of the manuscript; and in the decision to submit the paper for publication.

\section{Acknowledgements}

This work was supported by a grant from the National Natural Science Foundation of China (31400884, 81571317), the Beijing Municipal Science \& Technology Commission Grant (Z161100000216138), the Beijing Training Project for the Leading Talents in Science and Technology (Z151100000315020), CAS Key Laboratory of Mental Health, Institute of Psychology; and China Scholarship Council. 


\section{Click here to access/download \\ Supplementary Material
Supplementary Material 11_05_20.docx Supplementary Material
Supplementary Material 11_05_20.docx Supplementary Material
Supplementary Material 11_05_20.docx}

